\section{New osteoporosis strategies}

\section{By Brian Moy, Staff Writer}

A study by Japanese researchers suggests a pair of new strategies for treating osteoporosis: using iron chelation therapy and antagonizing PGC-1 $\beta$. Both approaches are geared at targeting regulators of mitochondrial biogenesis, an energy-generating process that osteoclasts use to fuel their bone-resorptive effects. In both cases, the challenge will be to specifically target only the activity of osteoclasts and not the bone-forming activity of osteoblasts.

Osteoporosis is caused by an imbalance in the activity of osteoclasts and osteoblasts. ${ }^{1}$ In a Nature Medicine paper, researchers led by principal investigator Kyoji Ikeda at the National Center for Geriatrics and Gerontology reported that mitochondrial biogenesis is regulated during osteoclast development by PGC-1 $\beta$ and iron uptake through the transferrin receptor (TFRC; TFR1).

The team found that knockdown of peroxisome proliferation-activated receptor- $\gamma$, coactivator $1 \beta$ (PPARGC1B; PGC-1 $\beta$ ) in bone marrow macrophages inhibited osteoclast differentiation compared with that seen in untreated control cells. In mice, knockdown of Pgc-1 $\beta$ led to greater bone mass than that seen in wild-type mice.

These findings, they wrote, "may provide a platform for the development of therapeutic strategies against various bone diseases with accelerated osteoclastic bone resorption," including osteoporosis.

Ikeda, head of the Department of Bone and Joint Disease at the National Center for Geriatrics and Gerontology, told SciBX, "Our findings also show that PGC- $1 \beta$ deficiency is manifest most prominently in osteoclasts." Thus, he said, "PGC- $1 \beta$ antagonism seems to be a rational approach to combat bone diseases without having serious side effects in other energy-requiring organs that are dependent upon mitochondrial oxidative energy metabolism."

Ikeda and colleagues are now screening for compounds that inhibit PGC-1 $\beta$ production or activity in osteoclasts.

The study also showed that iron uptake through TFRC promoted osteoclast differentiation and bone-resorbing activity. In ovariectomized estrogen-deficient mice, the iron chelator desferrioxamine inhibited tfrc-mediated bone resorption by mature osteoclasts and prevented bone loss compared with what was seen in vehicle-treated controls. $^{2}$
Thus, the authors said, "our data suggest a new explanation that iron overload leads to increased production and function of osteoclasts and may underlie the accelerated bone resorption observed in thalassemia patients."

Ikeda said iron chelators, such as Exjade, could be useful for the management of skeletal complications in thalassemia patients who have undergone repeated blood transfusions, which often result in iron overload.

Exjade deferasirox, an oral iron chelator from Novartis AG, is approved in the U.S. and EU to treat chronic iron overload due to blood transfusions.

\section{Target specificity}

Although the findings clearly point to new ways to treat osteoporosis, a key issue is whether PGC-1 $\beta$ and iron uptake play roles in the function of osteoblasts.

"We certainly don't want to inhibit bone formation by antagonizing PGC-1 $\beta$," said Clifford Rosen, senior scientist at the Maine Medical Center Research Institute. "While the strategies identified in the Nature Medicine paper are certainly promising, we need to further investigate exactly why bone formation was suppressed in these mice and further elucidate the role of PGC- $1 \beta$ in osteoblast function."

The authors of the Nature Medicine article suggested that the impaired bone formation seen in mice lacking Pgc- $1 \beta$ partly reflects the lack of functional Pgc-1 $\beta$ in osteoblasts.

Robert Brommage, associate director of metabolism research at Lexicon Pharmaceuticals Inc., was less convinced about the therapeutic potential of the strategies and suggested that both iron metabolism and PGC- $1 \beta$ "may have some pitfalls as drug targets for osteoporosis."

Brommage thinks modulating iron metabolism will have effects throughout the entire body, rather than only on osteoclasts. Regarding PGC- $1 \beta$, he said bone "may not be the only tissue affected by inhibition of PGC- $1 \beta$." Indeed, PGC- $1 \beta$ is highly expressed in several tissues characterized by high oxidative metabolism, including brown adipose tissue and skeletal and cardiac muscle. ${ }^{3}$

Through its large-scale knockout mouse program, Lexicon has identified an anti-resorptive target that "has a much stronger bone phenotype than PGC-1 $\beta$," according to Brommage. The company would not disclose the target or development plans.

Ikeda agreed that there might be some problems regarding the sensitivity of osteoclasts compared with that of osteoblasts to the inhibition of PGC-1 $\beta$. However, with additional research into the role of PGC-1 $\beta$ in osteoblasts, he thinks it will still be possible to develop drugs that inhibit the target satisfactorily in osteoclasts without exhibiting deleterious effects in osteoblasts.

Ikeda added that adverse effects due to iron chelation on osteoblasts are likely to be of little concern due to the fact that osteoblasts express 


\section{TARGETS \& MECHANISMS}

very little TFRC.

The Japanese researchers have filed a patent application in Japan covering PGC-1 $\beta$ and iron uptake through TFRC as methods for regulating osteoclastic bone resorption. The patent is available for licensing.

Moy, B. SciBX 2(10); doi:10.1038/scibx.2009.391

Published online March 12, 2009

\section{REFERENCES}

1. Teitelbaum, S.L. Science 289, 1504-1508 (2000)
2. Ishii, K. et al. Nat. Med.; published online March 1, 2009; doi:10.1038/nm.1910

Contact: Kyoji lkeda, National Center for Geriatrics and Gerontology, Aichi, Japan e-mail: kikeda@nils.go.jp

3. Lelliott, C.J. et al. PLoS Biol. 4, 2042-2056 (2006)

COMPANIES AND INSTITUTIONS MENTIONED

Lexicon Pharmaceuticals Inc. (NASDAQ:LXRX), The Woodlands, Texas Maine Medical Center Research Institute, Scarborough, Maine National Center for Geriatrics and Gerontology, Aichi, Japan Novartis AG (NYSE:NVS; SIX:NOVN), Basel, Switzerland 